\title{
Drug absorption through the skin: a mixed blessing
}

Skin is a barrier that keeps body water in and microorganisms and noxious chemicals out. Topical drugs, the mainstay of treatment in dermatology, are applied in the hope and expectation that any percutaneous absorption will be minimal and that systemic side effects will not occur. It is becoming apparent though that skin is not quite as impermeable as often believed. Undoubtedly, drugs can be absorbed through the skin and produce either unwanted or intended systemic effects.

\section{Mechanism of percutaneous absorption ${ }^{1}$}

The superficial layers of the epidermis, the stratum corneum, provide almost all the skin's barrier properties. (The basal epidermal layers and the underlying dermis are readily permeable - the often used term 'transdermal absorption' is inaccurate.) The stratum corneum is made up of layers of overlapping cell plates containing the fibrous protein keratin. Most drug absorption is transcellular: it is unlikely that noticeable absorption occurs between cells or through sweat pores and hair follicles. It is a passive diffusion process, the magnitude of which will depend on the integrity and efficacy of the epidermal barrier but which will be influenced by the drug itself. Drugs with low molecular weight (below 800 daltons) with a high water and lipid solubility show the greatest penetration. The vehicle that contains the applied drug is important. So too is the degree of hydration of the stratum corneum: occluding the epidermis increases its water content, enhancing drug absorption.

\section{Paediatric considerations}

Size. The smaller the child the larger the surface area relative to body weight. Absorption of drugs depends on the surface area exposed, whereas distribution, metabolism, and excretion relate more to weight. Percutaneous drug absorption is therefore more important in a very low birthweight infant than in an older child.

Integrity of the epidermis. Percutaneous absorption is greatly increased if the epidermal barrier is damaged or diseased. Young children with burns and infants with extensive skin disease (particularly eczema) are therefore at risk.

Maturity of the epidermis. The stratum corneum is well developed in the term infant and has similar barrier properties to those of a child or adult. It is poorly developed in preterm infants, particularly those with gestation less than 28 weeks. ${ }^{2}$ Such infants have a very high transepidermal water loss and readily absorb topically applied agents, sometimes with disastrous consequences. Immaturity of the stratum corneum is only temporary. The extrauterine environment triggers rapid maturation, so that by 2 or 3 weeks of age the skin has similar barrier properties to that of a term infant.

\section{Hazards of percutaneous absorption}

In 1886 Dr W Rayner, visiting medical officer at the Marylebone Workhouse, London, reported an outbreak of cyanosis in newborn infants. ${ }^{3} \mathrm{He}$ found an image of the workhouse stamp used for marking the nappies imprinted on the buttocks and vulva of one of the infants and correctly deduced that something in the ink (an aniline dye) was being absorbed to cause cyanosis (methaemoglobinaemia). His message went unheeded and at least nine further outbreaks of methaemoglobinaemia caused by absorption of aniline dyes have been reported, with considerable mortality.

Reports of other drugs and chemicals being absorbed through the skin and causing toxic effects are numerous. Most involve dermatological preparations and topical antiseptics, not surprisingly as such agents are often liberally applied to large areas of the skin of infants.

Dermatological preparations. Systemic effects from the absorption of topical steroids have often been reported. Most affected are young infants with extensive eczema treated with powerful fluorinated steroids. Effects range from adrenal suppression to reduced growth and frank Cushingoid features. A solution of $1 \%$ hydrocortisone does not seem to produce these effects and is therefore safer in the treatment of eczema in infants. Many other topical agents have been reported to be absorbed through the skin, causing toxic systemic effects. Perhaps the most notable is the aminoglycoside neomycin, which is never used systemically because of ototoxicity. It is often used in the form of a triple antibiotic aerosol spray (with polymixin and bacitracin), which if used liberally on damaged skin can be absorbed.

Topical antiseptics. Hexachlorophene was widely used as a topical antiseptic for the prevention of staphylococcal infection in the newborn. For 20 years it was common practice for infants to be 
bathed in it. Animal toxicology studies showed that central nervous system damage occurred, with a characteristic intramyelin oedema and vacuolation, but it was assumed that it was safe in the newborn infant because blood concentrations were so low. Toxicity was only reported in infants with damaged or diseased skin. When preterm infants were studied, however, it was clear that absorption was much greater, and brain lesions identical to those seen in poisoned animals were identified in 17 out of 248 infant autopsies. ${ }^{4}$ Common features of these 17 infants were prematurity and repeated exposure to hexachlorophene. Tragic confirmation that hexachlorophene is neurotoxic after percutaneous absorption came when a French manufacturer accidently marketed a batch of baby talcum powder that contained $6.3 \%$ hexachlorophene (it should have contained none). Altogether 204 infants were poisoned, with an illness characterised by a severe ulcerating nappy rash, fever, convulsions, and coma, of whom 36 died. ${ }^{5}$

Iodine and alcohol cause local and systemic effects, and although chlorhexidene is thought to be safe it is certainly absorbed through the immature skin. Poisoning from topical absorption of drugs and chemicals should always be borne in mind by staff who care for infants below 28 weeks' gestation. The skin is such a poor barrier that topical application is similar to oral administration. The very immature infants are usually sick and often die; tragedies such as have happened to older infants could easily be ascribed to natural illness and pass unnoticed.

\section{Uses of percutaneous absorption}

Drugs can be topically applied for local or systemic effect. $^{6}$

Local effect. The use of local anaesthetic cream is a welcome innovation. Usually, drugs like lignocaine are poorly absorbed and do not produce local anaesthesia. A new preparation, a eutectic mixture of $2.5 \%$ lignocaine and $2.5 \%$ prilocaine as an oil water emulsion, is absorbed appreciably when placed under an occlusive dressing. It produces very good local anaesthesia in children. The cream (EMLA cream, Astra Pharmaceuticals Ltd) is particularly useful for children who need a venepuncture. Disadvantages are that it has to be applied an hour in advance and that the cream under the occlusive dressing is rather messy and is expensive (about $£ 1$ a time), but these are outweighed by the pain children are spared. An alternative preparation suitable for the poorly developed skin of a preterm infant would be welcome in the neonatal intensive care unit.
Systemic effect. If a drug can exert its effects in minute dosage the small amount absorbed through the skin may be sufficient for systemic effect. A number of drugs can be administered to adults through the skin-namely, nitroglycerine for angina, hyoscine for travel sickness, clonidine for hypertension, and oestrogens for replacement therapy (only nitroglycerin is available in the United Kingdom). The drug delivery system is in the form of an adhesive patch, containing (from the outside to the skin surface) an occlusive backing, a reservoir of the drug, a microporous membrane, and an adhesive. The microporous membrane is less permeable to the drug than the skin and is therefore rate limiting, releasing the drug in a controlled way. Such a method of drug administration is not simply a curious gimmick. It is convenient, requiring less frequent dosage than oral administration, produces more predictable and constant blood concentrations, can be taken by vomiting patients, and can be removed at once. Unfortunately, most drugs given chronically to children (anticonvulsants, antibiotics, and bronchodilators) require too high a dose to be effective through the percutaneous route.

Where percutaneous treatment with drugs may have a part to play is in the treatment of the preterm infant. Small size and immature skin mean that a drug such as theophylline, which could not be given topically to a child, is readily absorbed and reaches therapeutic blood concentrations. ${ }^{7}$ Oral treatment with drugs in the small sick infant is unreliable and often contraindicated, while intravenous treatment requires skilled staff to establish and maintain it and is more hazardous and painful. Development of drug delivery systems that would enable preterm infants to be treated through the skin is an exciting possibility.

\section{References}

' Montagna W. Van Scott EJ. Stoughton RB, eds. Pharmacology and the skin. New York: Appleton-Century-Crofts, 1972.

2 Harpin VA. Rutter N. Barrier properties of the newborn infant's skin. J Pediatr 1983:102:419-25.

${ }^{3}$ Rayner W. Cyanosis in newly born children caused by aniline marking ink. $\mathrm{Br}$ Med $J$ 1886;i:294.

+ Shuman RM, Leech RW. Aluord EC. Neurotoxicity of hexachlorophene in the human: 1. A clinicopathologic study of 248 children. Pediatrics 1974:34:689-95

5 Martin-Bowyer G, Lebreton R. Toga M. Stolley PD, Lockhart J. Outbreak of accidental hexachlorophene poisoning in France. Lancet 1982;i:91-5.

- Shaw JE. Urquhart J. Transdermal drug absorption: a nuisance becomes an opportunity. Br Med J 1981:283:875-6.

7 Evans NJ, Rutter N, Hadgraft J, Parr G. Percutaneous administration of theophylline in the preterm infant. $J$ Pediatr 1985;107:307-11.

N RutTER

Department of Neonatal Medicine and Surgery, City Hospital, Nottingham NG7 $2 U H$ 